\title{
El internet y el comercio electrónico como desarrollo de las Pymes.
}

\section{The internet and e-commerce as a development of Pymes.}

Cristina Estefanía Acosta Rivera ${ }^{1}$, Adriana Roció Tintín Caiza ${ }^{2}$

\section{DOI: https://doi.org/10.33262/visionariodigital.v1i2.240}

\section{Resumen.}

Lo que sigue es una investigación para determinar cómo es el desarrollo del comercio electrónico y su interacción con los medios de comunicación y es un sistema digital personalizado y está a disposición de las personas ya través de la mejora de la tecnología ha sido de gran importancia a la educación en la actualidad.

El comercio electrónico tiene una gran acogida por la gente que necesita para vender productos o servicios a través de la web también tiene que ver con los medios de comunicación porque en el escenario del comercio electrónico no es tecnológico progreso.

Se trata de una nueva industria en la que una nueva plataforma, un nuevo medio y una nueva experiencia nos llevan a tener nuevas oportunidades de negocio.

Creaciones multimedia generalmente se basan en el contenido del archivo con gran atención al detalle, haciendo hincapié en los recursos expresivos más adecuados.

La motivación y el interés del destinatario del documento también promoverán elementos de la marca o una señal de que puede incluir sonidos o efectos de diversa índole en el momento adecuado.

Si la presentación multimedia permite al usuario actuar sobre la velocidad de secuencia o cualquier otro elemento progresar comercio electrónico nos da las herramientas necesarias para que nuestros productos y servicios se venden, o plantea preguntas, pruebas o alternativas que cambian su curso de multimedia de desarrollo, ya que puede decir, entonces describir multimedia, interactivos.

${ }^{1}$ Universidad Técnica de Ambato, FCADM, Ecuador, criss4546@hotmail.com ${ }^{2}$ Universidad Técnica de Ambato, FCADM, Ecuador, adriss2411@hotmail.com 
Las compras de bienes y servicios en línea o fuera de línea pueden ser atractiva para la instalación para llevar a cabo, sin embargo, es importante que los consumidores en línea toman precauciones para evitar ser víctimas de prácticas comerciales fraudulentas

Palabras Claves: Comercio electrónico, Multimedia, Hipermedia, Navegador.

\section{Abstract.}

What follows is an investigation to determine the development of multimedia and digital is a customized system that is available to people and thanks to of great importance to advancing education today.

It is a new experience, where the interaction with the media I radically different and where we have to learn how to use them.

It is a new industry where a new platform, a new medium and a new experience lead us to have new business opportunities.

Multimedia creations are usually based on file contents with great attention to detail, emphasizing by the most sophisticate expressivere sour cess particular a better understanding by the media. The motivation and interest of their cipi end of the document will al so encourage impact elements, among which may include sounds of various kinds at the right time. Also the organization of information can be improved using sophisticated models of navigation through the document.

Multimedia presentation allows the user to action these quences peedor any other element of their development, or raises questions, testing or alternatives hat modify its course, then describe as interactive multimedia. If he presentation is also constructed as a graph, so that you can

Navigate from one point to other following elements of link, then. It is a hypermedia creation. Hypermedia documents with channel features allow user interest through a route he is choosing at every moment. Thus access to the informations ought is easier.

Keywords : Development, Multimedia, Hipermedia, Browser.

\section{Introducción.}

En el presente trabajo analizaremos la importancia, la historia las ventajas, las desventajas del comercio electrónico y el desarrollo de la multimedia y desde su aparición hasta la actualidad siempre teniendo en cuenta que gracias a la multimedia se puede tener una visión amplia de la tecnología interaccionada con el comercio electrónico y el desarrollo de herramientas funcionales como son digitales entre otros.

Este trabajo se enfoca en analizar las ventajas de utilizar las herramientas del comercio electrónico y la multimedia para un aprendizaje de alta calidad 
Utilizado en la empresa sería un aporte muy importante ya que en la empresa se necesita innovar, para es muy significativamente utilizar herramientas del comercio electrónico y la interacción de la multimedia para que los productos del cliente tengan los resultados esperados y el cliente a su vez se sienta satisfecho con la calidad del producto gracias a la tecnología que disponemos en la actualidad.

También analizaremos la historia del comercio electrónico y de la multimedia como un ente transformador, de conceptos y de este a su vez como al pasar del tiempo tiene diferentes evoluciones.

Los programas multimedia tienen un gran aporte para la sociedad y para los estudiantes principalmente ya que estos tienen deberes consultas lecciones etc., tenemos que aprender más de la tecnología y aun mas de las herramientas del comercio electrónico.

Una de las herramientas tecnológicas que utilizamos hoy en día son los teléfonos la web y la computadora, para la utilización de estas es necesario tener un conocimiento de la multimedia y de la alta tecnología.

Está por demás decir que la comunicación basada en la aplicación de estas tecnologías cuyo dominio exige capacidades extremadamente desarrolladas.

Para lo cual la multimedia aparece cuando se pensaba utilizar el correo o el uso de la comunicación virtual y digital por ejemplo cuando hablamos de multimedia interactiva se puede decir que es cuando el usuario del proyecto puede controlar ciertos elementos y cuando deben controlarse.

La multimedia requiere cuando es necesario tener acceso a la información electrónica de cualquier tipo y mejora la comunicación entre individuos para tener un ente de información veraz y concisa.

Y presenta la información de diferentes formas que no son comunes a las que estamos utilizando en los negocios sirve para realizar presentaciones publicidad productos.

La multimedia y el comercio electrónico virtual se utiliza para la capacitación de diferentes conceptos de tecnologías es el medio más novedoso y comunicativo la manera de visualizar la multimedia es entenderlo como un medio de comunicación absolutamente interdisciplinario, donde la interacción entre el usuario y la multimedia es lo más importante sin duda alguna aunque el concepto multimedia va más allá de la tecnología estamos bien consientes que este ha podido surgir con el pasar de los años y es más rápido para una efectiva comunicación , y su base primordial es la utilización de tecnología .

El comercio electrónico necesita de la interacción de la multimedia, para su funcionamiento.

Métodos. 
Los métodos que se utilizó en este artículo o investigación es la investigación bibliográfica ya que nos basamos en los documentos o artículos de revistas o libros que de acuerdo a estos, fuimos recopilando información veraz y concisa aplicando estrategias de investigación proporcionadas anteriormente.

De acuerdo a la investigación que realizamos el método bibliográfico obtuvimos la información contenida en documentos que contiene la presente información.

Este método nos permitió seleccionar la información necesaria para realizar la conceptualización.

Gracias a este método tenemos una visión acerca de lo que queremos desarrollar.

También utilizamos la investigación científica porque lo hicimos a través de herramientas tecnológicas.

La presente investigación nos ayuda a mejorar el estudio porque nos permite establecer contacto con la realidad a fin de que la conozcamos mejor el desarrollo tecnológico. Constituye un estímulo para la actividad intelectual creadora. Ayuda a desarrollar una curiosidad creciente acerca de la solución de problemas, además, Contribuye al progreso de la lectura señala como realizar un avance tecnológico y la lectura crítica.

\section{Estado del arte.}

Lopez, P. C. (2005), Desde el punto de vista comunicaciones, podemos definir al Comercio Electronico como la entrega de informacion, productos, o servicios y pagos por medio de redes o cualquier otro medio electrónico.

El comercio electrónico consiste en realizar transacciones comerciales electrónicamente. Consiste en la transmisión electrónica de datos, incluidos texto, imágenes y vídeo. El comercio electrónico comprende actividades muy diversas, como comercio electrónico de bienes y servicios, suministro en línea de contenidos digitales, subastas, entre otros. El Comercio electrónico consiste en realizar electrónicamente transacciones comerciales; es cualquier actividad en la que las empresas y los consumidores interactúan y hacen negocios entre sí por medio de los medios electrónicos.

\section{Historia del Comercio Electrónico.}

La historia del Comercio electrónico es bastante similar a la de la Internet, el Internet surgió en los años 60 como proyecto del ARPA (Agencia de Investigación de proyectos avanzados de defensa). Respecto al comercio electrónico se suelen considerar en 4 generaciones.

Respecto a la Primera Generación: en el año de 1993 las grandes empresas perciben la importancia y comienzan a crear sus sitios web, primero de una manera en la que solo 
hablan de su negocio, posteriormente empezaron a realizar catálogos en la red, las páginas son estáticas y el modo de comunicación consistía en un formulario que contactaba a través del correo electrónico.

La segunda generación: Inmediatamente las empresas ya vieron la posibilidad de emplear páginas web para sus negocios surgen los centros comerciales virtuales, que consisten en una infraestructura de una tienda virtual e incluso rentaban espacios para otras tiendas que estuviesen interesadas en dar a conocer sus productos, en cuanto al medio de pago se realizaba a través de tarjetas electrónicas las cuales consisten en transferencias de dinero a través de una tarjeta.

Tercera Generación: se pretende automatizar el proceso de selección y el envío de datos acerca de los productos comprados, surgen las primeras implementaciones de bases de datos junto con aplicaciones web dinámicas y de fácil interacción con el usuario, surge la publicidad "el marketing en la red" y aparecen los primeros protocolos de pago seguro a través de las tarjetas electrónicas.

Cuarta Generación: el contenido ya es completamente dinámico generado a partir de una aplicación web a partir de datos suministrados por un sistema de base de datos, se cuida el diseño y aspecto del sitio empleando diseñadores gráficos especializados en su creación e informáticos para a programación del sitio web, se mejora la seguridad en el sitio y se implementan diversos nuevos mecanismos de control de seguridad.

Además de comprar y vender, las empresas realizan muchas otras actividades, que las mantienen en el negocio, por ejemplo, el vendedor de un producto debe identificar la demanda, promover su producto ante los compradores potenciales, aceptar pedidos, entregar su producto y apoyar el uso de su producto, facturar y recibir el pago de su producto. Y apoyar el uso de su producto por parte de sus clientes después de la venta.

De esta manera se han ido llevando a cabo distintas etapas en la historia del comercio electrónico y seguirá evolucionando al pasar de los años.

El comercio electrónico está en constante crecimiento, mencionó Marcos Pueyrredon, presidente del Instituto Latinoamericano de Comercio Electrónico (ILCE), en la conferencia internacional 'E-Commerce Day', que organizó la Cámara de Comercio de Guayaquil (CCG).

Pese a que esta actividad virtual en el país "se encuentra aún en pañales", Pueyrredon manifestó que este tipo de comercio "va creciendo muy rápido" en Ecuador.

"En 2010, en Latinoamérica cerramos con 35.000 millones de dólares, y de ese monto, más de 200 millones corresponden a Ecuador", indicó el presidente del ILCE, al tiempo de comentar que Brasil es el principal país de América Latina que mueve el $60 \%$ del comercio virtual. 
"Como sector, en los últimos tres años estamos creciendo en una tasa promedio del $50 \%$ en Latinoamérica, en que Ecuador está dentro de ese porcentaje. Y tenemos proyectados para los próximos años seguir por arriba de esa tasa", señaló.

Ante ello, el presidente de la CCG, Eduardo Peña, dijo que esta clase de iniciativas "empujarán" a que Ecuador sea parte de los países "en vanguardia del comercio electrónico".

Peña sustenta a esta nueva actividad comercial como "un trípode", en que explicó que una de sus tres patas es la banda ancha de Internet, en el que reconoció el esfuerzo que ha hecho el Gobierno para "dotarnos mejor de este servicio".

\section{Discusión.}

\section{Definición de comercio electrónico.}

Según los autores Balado, E. S. (2005); Electrónico, S. e. (2010); Gariboldi, G. (1999); Julian Briz, I. L. (2001); Lopez, P. C. (2010); Nos hablan acerca de que el Comercio Electronico es una moderna metodología que da respuesta a varias necesidades de empresas y consumidores, en la mayoría de las veces hace referencia a la venta de productos por Internet.

También se considera aspectos muy fundamentales como reducir costes, mejorar la calidad de productos y servicios, acortar el tiempo de entrega o mejorar la comunicación con el cliente.

Podemos definir el comercio electrónico como cualquier forma de transacción comercial, no sólo comprar y vender en la que las partes interactúan electrónicamente en lugar de por intercambio o contacto físico o de la misma manera puedes ser directamente. También podríamos hablar de negocios electrónicos, que es más general y reservar el término comercio para las compras y ventas por Internet.

Además también podemos hablar que el Comercio Electronico radica en la compra y venta de mercancias y servicio de acuerdo a la necesidad dl cliente para dar mejor satifaccion en mopjeores condiciones, y de esta manera se sientan a gusto con e producto o servicio.

Asmismo podemos decir que el Comercio Electronico es una actividad economica y social que ala vez reside en elintercambio de bienes y servicios las mismas que pueden ser para su uso, para su venta o para su tranformacion.

\section{Historia.}

para los autores, Perez, G R. (2010); Kleinrock, L. (1964); Lamara, M. J. (2001); Julian Briz, I. L. (2001); Lopez, P. C. (2010); Nos hablan que a principio de los años 1970, asomaron las primeras relaciones comerciales que utilizaban una computadora para transmitir datos, tales como órdenes de compra y facturas. 
A mediados de 1980, con el apoyo de la televisión, nació una nueva forma de venta por catálogo, también llamada venta directa, de esta manera, los productos son publicados con mayor realismo, y pueden ser presentados sobresaliendo sus características.

En 1995 los países integrantes del gran mundo crearon la iniciativa un mercado global para pymes, con el plan de acelerar el uso del comercio electrónico entre las empresas de todo el mundo, durante el cual se creó el portal pionero en idioma español.

Asimismo nos dicen que el comercio electrónico es bastante similar como la de internet, ya que van de la mano con la aparición de la misma.

Estos autores nos dicen que de acuerdo a la necesidad del comercio electrónico se origina de la demanda de las empresas y de la administración, para crear un excelente uso de la informática e investigar una mejor forma de aplicar las nuevas tecnologías para así mejorar la interrelación entre cliente y proveedor.

Podemos reafirmar que el año que nació como tal el comercio electrónico fue hacia el año de 1920 en los estados unidos dónde apareció la venta por catálogo.

Este nuevo método de comercialización fue una gran revolución en ese momento, ya que fue la primera vez que se podía obtener sin antes ver el producto.

Asimismo, podemos hablar que la venta por catálogo trabajaba mediante fotos ilustrativas del producto.

La verdadera mejoría que posibilitaba este sistema comercial era que se podía vender en zonas rurales difíciles de acceder debido a diferentes circunstancias.

Igualmente sabemos que esta actividad nació de una necesidad de los hombres para ser autosuficientes.

El intercambio de bienes y servicios es un primer síntoma de la evolución, sin este intercambio no se podría asegurar el mantenimiento ni el desarrollo de los seres humanos.

En la actualidad la historia del comercio ha ido evolucionando, en cierta parte de una forma pausada y lenta

Lo que podemos ver que ahora como comercio electrónico es la última etapa a la cual hemos llegado de este sucesivo desarrollo.

\section{Tipos de comercio electrónico.}

Según los autores Aguila, R. (2005). Balseca, A. (2003) Gonzales, R. (1999) Hidalgo,O.

Peralta, J. F. (2001) nos dicen que el comercio electronico se clasifica en.

\section{Business to Business (B2B)}


Comercio realizado entre proveedores y clientes empresariales, o sea,de empresa a empresa.

\section{Business to Consumer o Business to Customer (B2C)}

Es el comercio entre la empresa productora, vendedora o prestadora de servicios y el consumidor final.

\section{Consumer to consumer $(\mathrm{C2C})$}

Comercio electrónico entre usuarios particulares de Internet. La comercialización de bienes o servicios no involucra a productores y sí a un consumidor final con otro consumidor final, sin intermediarios.

\section{Goverment to Consumers (G2C)}

Comercio entre gobiernos, provincial, federal o municipal y consumidor, por ejemplo, el Pago de impuestos, multas y tarifas públicas.

\section{Government to Business (G2B)}

Negocios entre gobierno y empresas, por ejemplo: las compras del Estado a través de internet por medio de licitaciones, concurso de precios, etc.

\section{Intermediario de la confianza}

Se entiende por este término a una entidad que crea confianza entre el comprador y el vendedor

\section{Informe diario}

Se concentran en formar numerosas alianzas, mantener un amplio contenido y promocionar sus sitios web entre los compradores.

\section{Escaparate de E-Commerce}

Aquella entidad económica en la que se desarrollan actividades comerciales utilizando canales tradicionales y digitales

Los tipos de comercio electrónico tienen en gran medida una parte de la multimedia ya que sin ella no podrían ser ejecutados debido a la gran información que estos contienen tienen a ser utilizados como herramienta primordial en un negocio en una pequeña empresa para solucionar diferentes problemas de la empresa y por ende a tratar asuntos netamente de negocios

Los tipos de comercio electrónico podrán ser de cierta manera unas pequeñas pautas para llegar al objetivo en un periodo determinado. 


\section{Ventajas y desventajas del comercio electrónico.}

Según los autores Aguila, R. (2005). Balseca, A. (2003) Gonzales, R. (1999). Hidalgo.

Peralta, J. F. (2001 nos manifiestan algunas ventajas y desventajas de coercio electronico

Permite establecer un nuevo canal de comunicación y conseguir la "interacción" entre empresas de distribución o venta y fabricantes o productores, Ofrece la posibilidad de crear compañías totalmente digitales, es decir, virtuales, y no es imprescindible disponer de un espacio físico.

Mejora el posicionamiento de la compañía, pero para eso debemos cultivar la marca y trabajarla a diario para conseguir, con constancia y paciencia, una identidad digital.

Favorece y modifica la relación que se establece entre el fabricante, el distribuidor y los consumidores, impulsando un flujo de información entre todos, que permite la reorientación del producto.

- Realizar mejor negociación con el vendedor.

- Comodidad en la adquisición del bien o producto

- Elimina obligaciones con trabajadores por los contratos.

- Costos de los distribuidores.

- Elimina las perdidas por mercancía robada.

- Elimina días muertos por causas de huelga.

- Genera mayores ganancias por venta unitaria de un producto.

- Menos inversión en los presupuestos publicitarios.

- Reducción de precios por el bajo coste del uso de Internet en comparación con otros medios de promoción, lo cual implica mayor competitividad.

\section{Desventajas .}

- No existe cercanía entre el vendedor y el comprador para proceder con una queja del producto.

- Cobro o poder hacer valida la garantía del producto comercializado.

- Se pierde la capacidad de visualización del producto en comercialización o conocimiento físico del producto.

- Aunque ha avanzado mucho el comercio electrónico, todavía no hay una transmisión de datos segura el $100 \%$.

Las ventajas es que es una forma de transacción rápida y es más barata y no lleva mucho tiempo para poder realizar es decir nos ahorra tiempo y dinero.

\section{Historia de internet.}


Para los autores (Atelin, P. 2000); (Gonzales, A. 2002); (Jiménez, I. 2005); (Tanenbaum, A.S. 2000); (Cervera, M.G. 2001), manifiestan que la Internet nació en Estados Unidos, en 1960 crea una red exclusivamente militar, en plena guerra fría, con el objetivo de que, en el caso de un ataque ruso, se pudiera tener acceso a la información militar desde cualquier punto del país.

Internet se inició como un proyecto de defensa de los Estados Unidos. A finales de los años 60, la ARPA (Agencia de Proyectos de Investigación Avanzados) del Departamento de Defensa definió el protocolo TCP/IP. La idea era garantizar mediante este sistema la comunicación entre lugares alejados en caso de ataque nuclear.

En Agosto de 1969 ARPANET se convierte en una red y es considerada la precursora de Internet. Fue desarrollado y demostrado por primera vez en 1972. El TCP / IP es la base del Internet que sirve para enlazar computadoras que utilizan diferentes sistemas operativos, incluyendo PC, y minicomputadoras. Ahora el TCP/IP sirve para garantizar la transmisión de los paquetes de información entre lugares remotos, siguiendo cualquier ruta disponible.

En 1975, ARPANET comenzó a funcionar como red, sirviendo como base para unir centros de investigación militares y universidades, y se trabajó en desarrollar protocolos más avanzados para diferentes tipos de ordenadores y cuestiones específicas. Actualmente, ARPANET es una red y es considerada la precursora de Internet.

En 1983 se adoptó el TCP/IP como estándar principal para todas las comunicaciones, y en 1990 desapareció ARPANET para dar paso junto a otras redes TCP/IP a Internet. Por aquel entonces también comenzaron a operar organizaciones privadas en la Red.

Poco a poco, los fabricantes de ordenadores personales y redes han incorporado el TCP/IP a sus sistemas operativos, de modo que en la actualidad cualquier equipo está listo para conectarse a Internet.

Internet une muchas redes, Se calcula que en la actualidad hay varios miles de redes de todos los tamaños conectadas a Internet.

Se puede considerar que la Internet se inició en torno al año 1969, cuando el departamento de defensa de los Estados Unidos desarrollo ARPANET, una red de ordenadores creada durante la guerra fría cuyo objetivo era eliminar la dependencia de un ordenador central, y así hacer mucho menos vulnerable las comunicaciones militares norteamericanas.

Luego se conectaron tres computadoras de diferentes universidades y también algunas personas desde sus casas. Fue entonces cuando se empezó a extender Internet por los demás países del mundo, abriendo un canal de comunicaciones entre Europa y EE.UU. 
Tanto el protocolo TCP / IP como el control de transmisión fueron desarrollados a partir de 1973, por el departamento de defensa norteamericano.

Cuando en los 1980 la red dejó de tener interés militar, paso a otras agencias que ven en ella interés científico.

Desarrollo de las Pymes

Las pequeñas y medianas empresas se enfrentan a muchos problemas para poder mantenerse en el mercado, aproximadamente se puede establecer que el $80 \%$ cierra antes de cumplir los dos años de haber sido lanzada al mercado.

Uno de los factores que intervienen para el fracaso es la falta de innovación tecnológica o el cambio o la poca publicidad existente en la nueva empresa el internet juegan un papel fundamental para que estas pequeñas empresas tengan el éxito.

Si bien una Pyme no cuenta con los recursos necesarios como implementar estrategias de mercado con una capacitación constante en la tecnología por medio del internet, como por ejemplo la publicidad por internet donde se incluye también el comercio electrónico

Como fuente de ingresos para la PYMES es darse a conocer por medio del internet para atraer más clientes para las pequeñas y grandes empresas su costo es significativamente bajo en comparación con medios tradicionales.

El internet es el medio más utilizado por los consumidores para la compra de bienes y servicios.

Cabe resaltar que los de internet pasan ala mayor parte del tiempo en las redes sociales, por lo que es necesario la utilización de diferentes páginas web para la promoción de un producto o servicio. Es en ese momento donde llega a tomar posición el comercio electrónico mediante la creación de distintas páginas web que tenga servicios múltiples para dar a conocer el bien o servicio.

Estos son algunos de los beneficios que una PYME tiene al invertir en publicidad por Internet:

- Mayor eficiencia a menor costo.

- Mayor alcance: Nuestra comunicación puede llegar a personas de todo el mundo.

- Interacción con el cliente: podemos conocer lo que opinan de nosotros y lo que busca o necesita.

- Se proyecta una buena imagen de marca. 
- Permite segmentar de mejor manera el mercado para dirigir nuestra publicidad a personas que tiene el perfil para interesarse en el producto que ofrecemos.

- Medición: Los resultados se pueden medir fácilmente.

La debilidad de las PYMES está relacionada con la falta de liquidez sobre todo para apoyar a los pequeños negocios.

Las fortalezas se definen como aquellas que se distinguen de la competencia por ejemplo la capacidad de formar una empresa a largo plazo con productos innovadores.

El internet como parte del desarrollo de las PYMES y los factores que influyen para el logro de los objetivos de las pequeñas y grandes empresas son muy influyentes entre las cuales tenemos

- Uso común de computadoras e internet por parte de la mayoría de empresas.

- Presupuesto para la inversión de hardware y software.

- Apoyo técnico para mejorar la productividad

- Uso de internet para la búsqueda de comercio en todas sus formas.

Las debilidades representan aquellos aspectos de las empresas que las hacen vulnerables ante la competencia, pérdida de posición en el mercado y rentabilidad reducida. Para las PYMES, en el caso de estudio, las debilidades están dadas por los siguientes factores:

- Uso de software no autorizado

- Falta de apoyo técnico por parte de las empresas proveedoras del software

- Desconocimiento sobre la importancia del uso de TICs para mejorar el desempeño

- Tamaño de la empresa Falta de destrezas para el manejo de TICs.

Las redes sociales se han convertido en un fenómeno global que determina dinámicas en el ámbito de cambio en el ámbito social profesional y económico.

La comunicación esta recóndito en el desarrollo de las PYMES, lo que se puede decir es que el funcionamiento de las redes sociales y del internet está en el consumidor y la persona quien emite el bien o servicio a través de una PYME.

La comunicación que las empresas tengan con su mercado meta objetivo puede lograrse alcanzando los objetivos corporativos.

Un eje transversal para la gestión del internet por parte de las organizaciones y estilos comunicacionales, basados en escuchas activas de su público. 
El crecimiento de los avances tecnológicos en el mundo actual y en países en vías de desarrollo ha aumentado por tanto en los negocios virtuales son un hecho las personas han optado por recurrir, a este medio por sus grandes ventajas la PYMES de Ecuador se enfrentan este importante reto de expandirse a nuevos mercados que permita optimizar sus recursos haciendo estas empresas ,negocios competitivos no solamente a nivel nacional sino a nivel internacional mediante una herramienta importante como el internet que permite el ingreso a mercados electrónicos considerados como negocios que abaratan costos ,y elimina los intermediarios .

El comercio electrónico es considerado como el futuro del comercio de las PYMES del Ecuador no pueden estar excluidas en tanto que otros países están maximizando su actividad emprendedora.

Las pequeñas y medianas empresas en Ecuador, son las unidades productivas con mayor productividad de participación en la economía es muy importante el desarrollo de políticas e innovación tecnológica para mantener la competitividad, permitiendo que estas empresas llegar con productos a lugares inaccesibles.

\section{Resultados.}

En base a la investigación que hemos realizado debido a cada tema se pretendió analizar y conocer cada uno de los beneficios su importancia sus tipos en si todo lo relacionado al tema de la Internet y el Comercio Electrónico como Desarrollo de las Pymes, ya que es un tema de carácter importante en el ámbito laboral, sobre todo como una metodología de enseñanza para los empresarios de hoy en día, es por esto que esta investigación resultó importante dándonos como resultado conceptos claros y concisos y sobre todo nuevos conocimientos adquiridos sobre el tema.

\section{Metodología de la investigación:}

Responde a la interrogante :¿Cómo y con que a investigar?. Por lo tanto se relaciona con el tiempo de investigación, la investigación, la población y muestra, las técnicas de recolección de información ,el procesamiento y análisis de los datos recogidos .

\section{Tipo de Investigación:}

La investigación estará de acuerdo a la información que se requiera y pude ser de campo, bibliográfica y experimental.

\section{Población y Muestra}

La muestra es una parte de la población en la que se aplicara técnicamente los instrumentos para obtener los datos .

Técnicas de Recolección de Datos: 
La recolección de datos consiste en diseñar las técnicas y los instrumentos que se aplicaran de acuerdo a lo que se investiga.

El plan de recolección de información debe hacerse mediante técnicas como: encuestas, entrevistas, observaciones, lecturas científicas.

\section{Procesamiento de los datos}

- Revisión critica de la información recogida

- Tabulación de datos

- Elaboración de cuadros y representaciones graficas estadísticas

\section{Análisis e Interpretación de Resultados}

Análisis de los resultados estadísticos.

A continuación, hemos elaborado una encuesta dirigida a las personas para conocer más acerca del tema.

Realizamos la tabulación de datos y posteriormente hemos procedido a interpretar los resultados.

\section{$\underline{\text { Análisis de datos }}$}

Gráfico 1.- ¿Cuánto a menudo utiliza el internet?

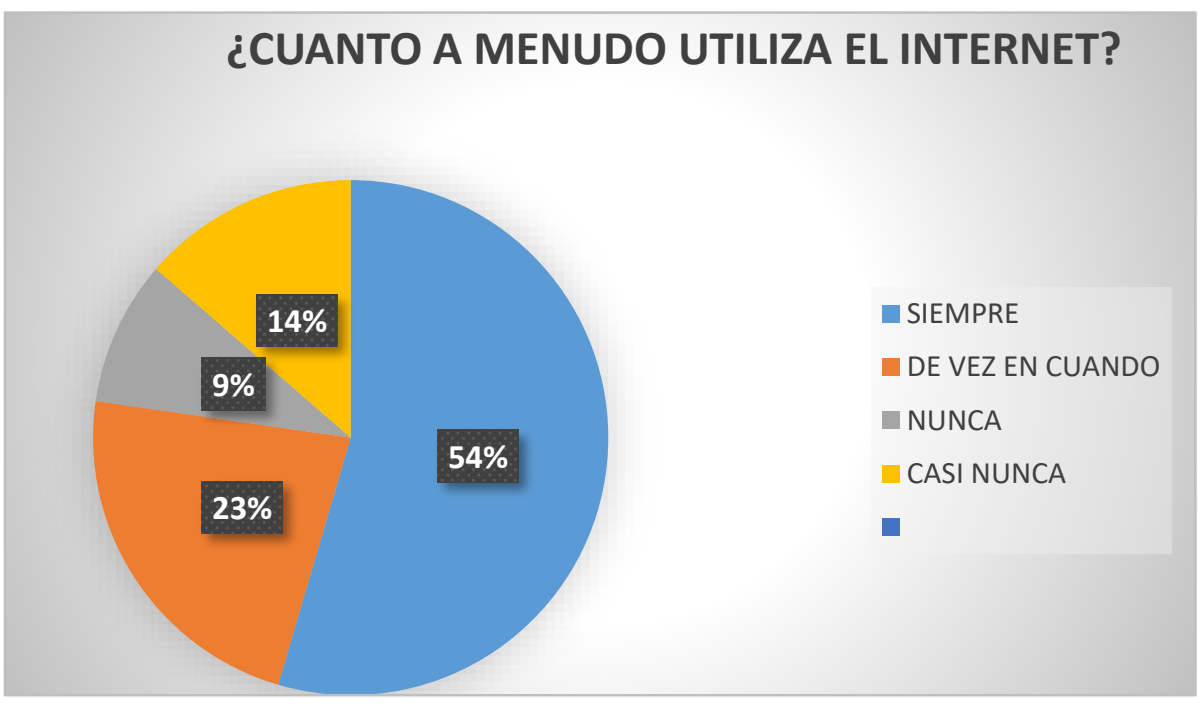

Elaborado por: Grupo de investigación.

Interpretación:

Del $100 \%$ de las personas encuestadas el $54 \%$ de las personas nos dicen que siempre utilizan el internet el $23 \%$ nos indican que utilizan de vez en cuando, el 14\% señala que casi nunca utilizan el internet, y el $9 \%$ manifiestan que nunca han utilizado el internet, podemos decir que lo que tiene más relevancia es el 54\% de las personas encuestadas que manifiestan que utilizan internet siempre. 
Gráfico 2.- el internet como herramienta del comercio electrónico es de gran ayuda en los negocios en la actualidad

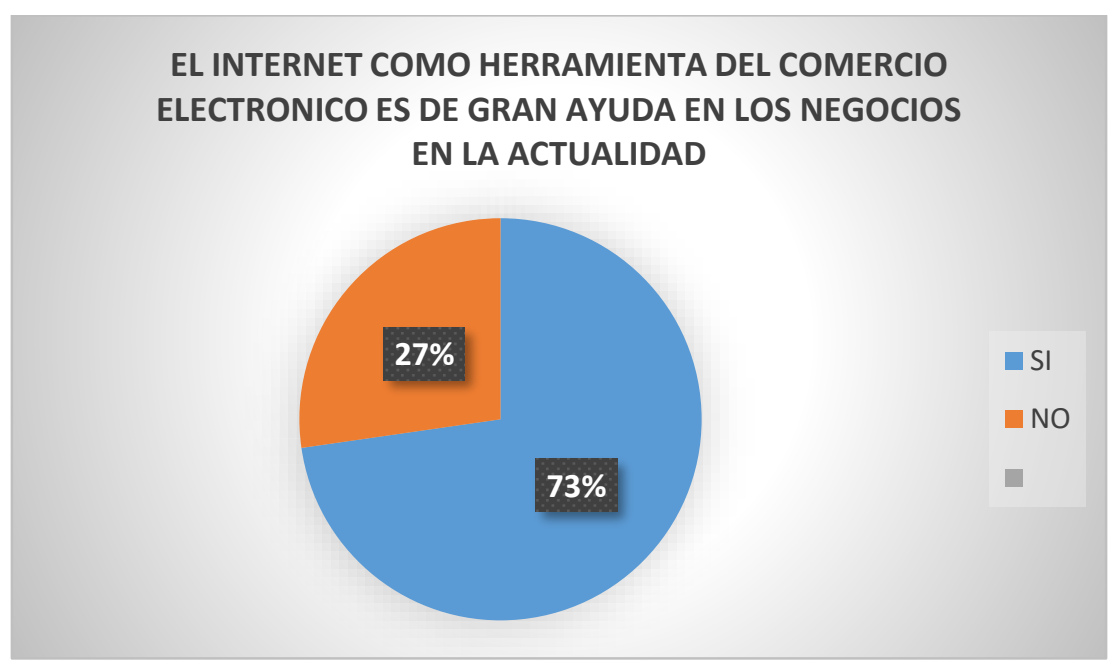

Elaborado por: Grupo de investigación.

Interpretación:

Del $100 \%$ de las personas encuestadas nos indican que el $73 \%$ de las personas piensan que el internet como herramienta del comercio electrónico es de gran ayuda en los negocios en la actualidad, y el $27 \%$ manifiestan lo contrario. Lo que se puede analizar es que hay más relevancia en que las personas dicen que si es importante el internet en los negocios de la actualidad.

Gráfico 3.- Que actividades realizas en internet.

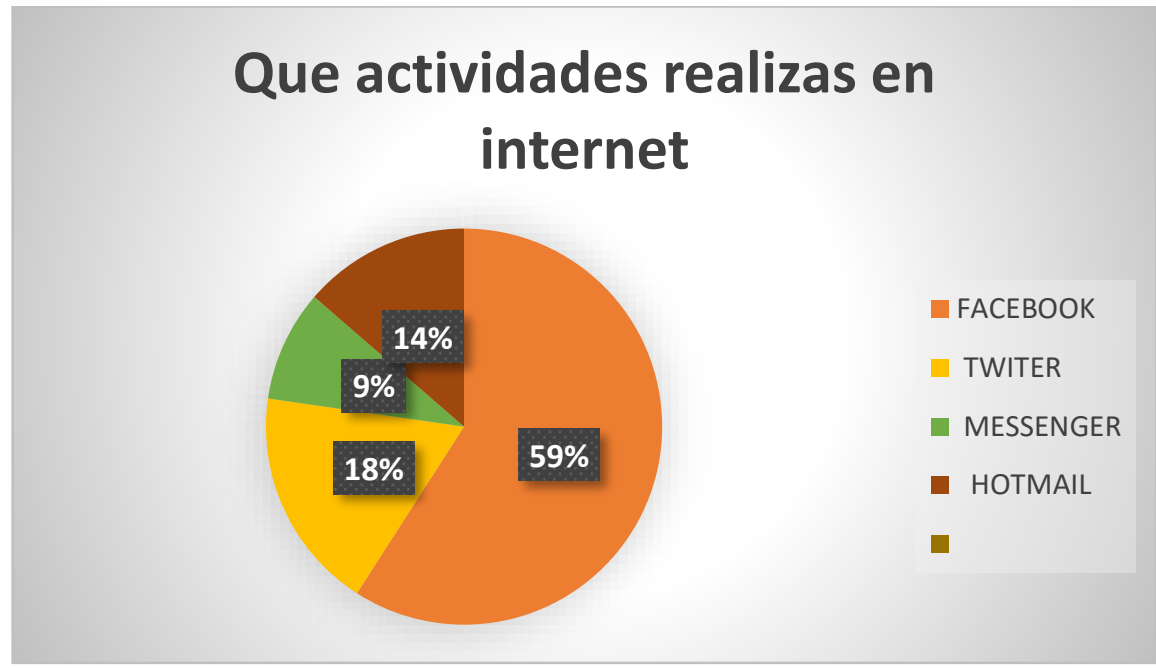

Elaborado por: Grupo de investigación. 
Interpretación.

Del 100\% de las personas encuestadas el 59\% utiliza Facebook, el 18\% utiliza twitter, el 14\% utiliza Hotmail, el 9\% utiliza Messenger podemos decir que lo que más relevancia tiene es que las personas mayormente utilizan el Facebook,

Gráfico 4.- ¿Qué actividades del mundo real ha disminuido por utilizar internet ?

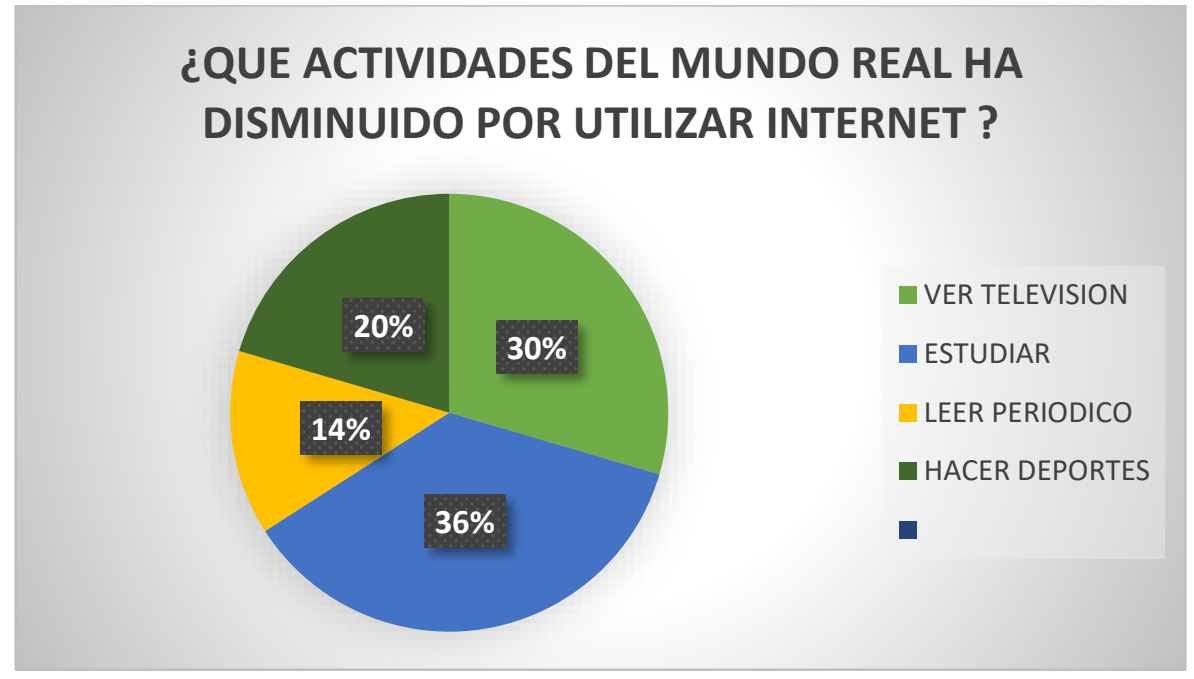

Elaborado por: Grupo de investigación.

Interpretación:

Del $100 \%$ de las personas encuestadas el $36 \%$ nos manifiesta que por utilizar internet a disminuido el estudiar, el $30 \%$ indica que a disminuido el ver televisión ,el $20 \%$ recalca que a disminuido el hacer deportes y el 14\% dice que ha disminuido leer periódico.

Gráfico 1.- Cuanto tiempo te conectas a internet a la semana.

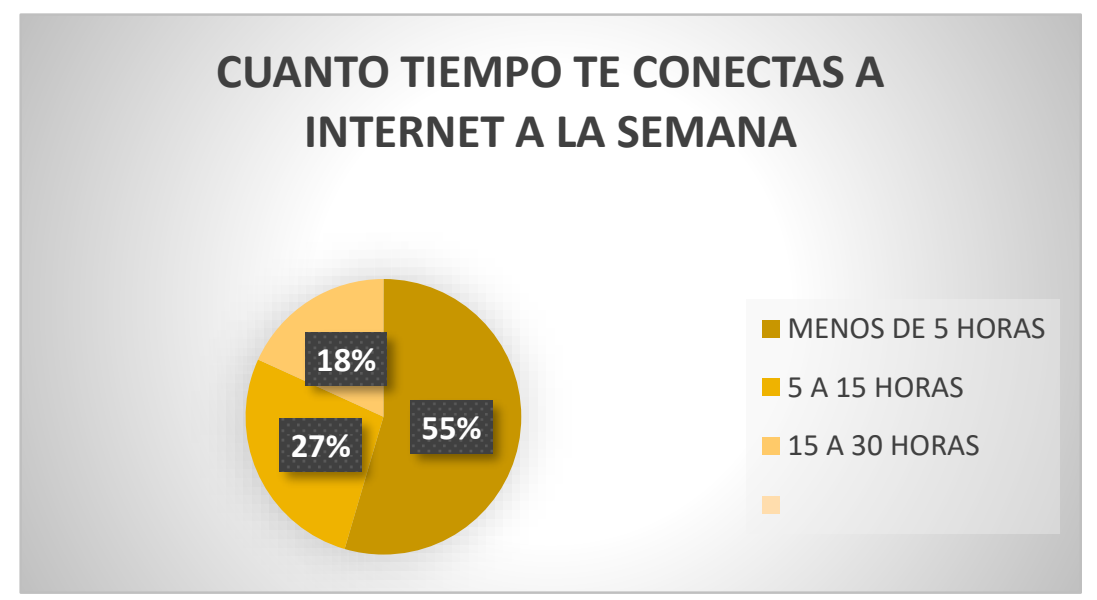

Elaborado por: Grupo de investigación. 
Interpretación:

Del $100 \%$ de las personas encuestada el $55 \%$ nos dicen que utiliza el internet menos de 5 horas el $27 \%$ utiliza de 5 a 15 horas ,18\% utiliza de 15 a 30 horas lo que se puede analizar es lo que mas dominio tiene es que las personas utilizan menos de 5 horas.

Análisis general.

De acuerdo a la interpretación de los resultados podemos manifestar que mas del 50\% esta de acurdo que la utilización del internet es fundamental para el desarrollo de las pymes.

\section{Conclusiones}

- El estudio del comercio electrónico y de las herramientas del internet en la actualidad es de gran importancia, ya que establece el éxito y buen trabajo de las diferentes actividades dentro de las Pymes, educación entre otros; por lo cual es importante conocer su funcionamiento en las diferentes áreas empresariales, es un medio que integra texto, imagen, grafico, video, para servicio del usuario.

- Como es la tecnología avanza constantemente y de esta manera ayuda al conocimiento y desarrollo de las Pymes y del comercio electrónico que es un medio de carácter importante y sirve de mucha ayuda para docentes y estudiantes personas y en los negocios en todo nivel.

- El comercio electrónico está ligado a la unión de herramientas del internet para que pueda funcionar correctamente y tenga los resultados esperados y se logre la venta de un bien o servicio para que se pueda tener buenos efectos en la empresa.

\section{Bibliografía}

(Barona, 2010)

Baran, P. (1994). Redes y Comunicaciones .

Galiano, G. S. (2014). Estudio comparativo de servidores multimedia. Mexico.

Jaramollo, I. D. (2012). Método y conocimiento metodología de la investigación. Mexico. 
Kleinrock, L. (1964). Flujo de Inormacion.

1.KLEINROCK. (1976). Aplicaciones Informaticas . New York.

Lopez, P. F. (2012). La sociedad del Conocimiento génesis de una revolución 3.0. Mexico.

Pelegrino, T. G. (2014). Multimedia educativa para la Enseñanza de la informática. Mexico.

R. Kahn. (1972). Principios para la comunicacion.

Ramirez, R. D. (2006). Método y conocimiento: metodología de la investigación. Mexico.

Romero, J. G. (2005). Los recursos informáticos en la educación del alumnado . Mexico.

Ruiz, M. (2002). Multimedia.

V. G. Cerf y R. E. Kahn. (1974). Protocolo para la intercnexion de redes.

Baran, P. (1994). Redes y Comunicaciones.

Galiano, G. S. (2014). Estudio comparativo de servidores multimedia. Mexico.

Jaramollo, I. D. (2012). Método y conocimiento metodología de la investigación. Mexico.

Kleinrock, L. (1964). Flujo de Inormacion.

1.KLEINROCK. (1976). Aplicaciones Informaticas . New York.

Lopez, P. F. (2012). La sociedad del Conocimiento génesis de una revolución 3.0. Mexico.

Pelegrino, T. G. (2014). Multimedia educativa para la Enseñanza de la informática. Mexico.

R. Kahn. (1972). Principios para la comunicacion.

Ramirez, R. D. (2006). Método y conocimiento: metodología de la investigación. Mexico.

Romero, J. G. (2005). Los recursos informáticos en la educación del alumnado . Mexico.

Ruiz, M. (2002). Multimedia.

V. G. Cerf y R. E. Kahn. (1974). Protocolo para la intercnexion de redes. 
Baran, P. (1994). Redes y Comunicaciones .

Galiano, G. S. (2014). Estudio comparativo de servidores multimedia. Mexico.

Jaramollo, I. D. (2012). Método y conocimiento metodología de la investigación. Mexico.

Kleinrock, L. (1964). Flujo de Inormacion.

1.KLEINROCK. (1976). Aplicaciones Informaticas . New York.

Lopez, P. F. (2012). La sociedad del Conocimiento génesis de una revolución 3.0. Mexico.

Pelegrino, T. G. (2014). Multimedia educativa para la Enseñanza de la informática. Mexico.

R. Kahn. (1972). Principios para la comunicacion.

Ramirez, R. D. (2006). Método y conocimiento: metodología de la investigación. Mexico.

Romero, J. G. (2005). Los recursos informáticos en la educación del alumnado . Mexico.

Ruiz, M. (2002). Multimedia.

V. G. Cerf y R. E. Kahn. (1974). Protocolo para la intercnexion de redes.

Baran, P. (1994). Redes y Comunicaciones.

Galiano, G. S. (2014). Estudio comparativo de servidores multimedia. Mexico.

Jaramollo, I. D. (2012). Método y conocimiento metodología de la investigación. Mexico.

Kleinrock, L. (1964). Flujo de Inormacion.

1.KLEINROCK. (1976). Aplicaciones Informaticas . New York.

Lopez, P. F. (2012). La sociedad del Conocimiento génesis de una revolución 3.0. Mexico.

Pelegrino, T. G. (2014). Multimedia educativa para la Enseñanza de la informática. Mexico.

R. Kahn. (1972). Principios para la comunicacion.

Ramirez, R. D. (2006). Método y conocimiento: metodología de la investigación. Mexico. 
Romero, J. G. (2005). Los recursos informáticos en la educación del alumnado . Mexico.

Ruiz, M. (2002). Multimedia.

V. G. Cerf y R. E. Kahn. (1974). Protocolo para la intercnexion de redes.

\section{Para citar el artículo indexado.}

Acosta C., Tintín A. (2017). El internet y el comercio electrónico como desarrollo de las Pymes..Revista electrónica Visionario Digital 1(1), 19-38. Recuperado desde: http://cienciadigital.org/revistacienciadigital2/index.php/VisionarioDigital/article/view/ $240 / 534$

\section{Ciencia}

El artículo que se publica es de exclusiva responsabilidad de los autores y no necesariamente reflejan el pensamiento de la Revista Ciencia Digital.

El articulo queda en propiedad de la revista y, por tanto, su publicación parcial y/o total en otro medio tiene que ser autorizado por el director de la Revista Ciencia Digital 